\title{
Stimulated Emission Depletion Studies of Molecular Probe Dynamics.
}

\author{
R J Marsh, N D Leonczek, D A Armoogum, E M Monge and A J Bain ${ }^{+}$ \\ Department of Physics \& Astronomy, University College London, Gower Street, London WC1E \\ 6BT, UK \\ ${ }^{+}$Corresponding Author.
}

\begin{abstract}
Stimulated emission depletion (STED) population and polarisation dynamics are used to determine $\left\langle\alpha_{40}\right\rangle$ the degree of hexadecapole alignment created in ensembles of rhodamine $6 \mathrm{G}$ molecules in solution following two-photon excitation. Hexadecapole molecular alignment is an unavoidable consequence of two-photon excitation but is not observed in spontaneous emission. For a single element diagonal transition tensor measurements of the fluorescence anisotropy $R(t)$ in systems undergoing small step isotropic rotational diffusion can in principle be used to determine $\left\langle\alpha_{40}\right\rangle$. STED measurements of rhodamine $6 \mathrm{G}$ yield a value for $\left\langle\alpha_{40}\right\rangle$ that is larger than that predicted for a single element transition tensor $\left(S_{X X}\right)$. Recent work in our laboratory indicates that whilst $S_{X X}$ is dominant $S_{Y Y}, S_{X Y}$ and $S_{Y X}$ are finite, measurements of $\left\langle\alpha_{40}\right\rangle$ appear to be a sensitive probe of the structure of the two-photon transition tensor. STED and fluorescence anisotropy measurements are extended to Rhodamine $6 \mathrm{G}$ in the isotropic phase of 5CB a system where small step isotropic rotational relaxation does not take place. Here the values of $\left\langle\alpha_{40}\right\rangle$ are considerably larger. These results are discussed in terms of the initial hexadecapole alignment and $\left\langle\alpha_{40}\right\rangle$ relaxation dynamics in a restricted geometry.
\end{abstract}

Key words: Stimulated emission depletion, two-photon fluorescence, molecular alignment, polarisation, transition tensor.

\section{INTRODUCTION}

Stimulated emission depletion (STED) of excited states has proved to be both a valuable tool in high resolution molecular spectroscopy ${ }^{1}$, in time resolved spectroscopy as a means of orientational photoselection ${ }^{2}$ and in the study of ultrafast vibrational relaxation within electronic ground states ${ }^{3}$. There has been considerable interest in the use of single photon STED in fluorescence microscopy ${ }^{4}$ where sub wavelength image resolution has been recently demonstrated ${ }^{5}$. Recent work in our laboratory ${ }^{6-10}$ has demonstrated the feasibility of performing STED in two-photon excited states. In this work femtosecond near infra red $(800 \mathrm{~nm})$ two-photon excitation (PUMP) is followed by visible picosecond stimulated emission depletion (DUMP) of the excited state. Time-resolved fluorescence detection is employed to determine changes in excited state population and alignment following STED together with the measurement of stimulated emission cross-sections and ground state vibrational relaxation times. Measurements of fluorescence anisotropy changes induced by STED are sensitive to higher degrees of excited state order and as such can be used to circumvent the constraints of single photon emission which are sensitive solely to molecular alignment (quadrupolar order). In this paper we determine absolute values for the normally hidden degrees of hexadecapolar molecular alignment created by two-photon excitation of rhodamine $6 \mathrm{G}$ in ethylene glycol and the isotropic phase of 5CB.

Nanophotonic Materials and Systems II, edited by Zeno Gaburro, Stefano Cabrini,

Proceedings of SPIE Vol. 5925 (SPIE, Bellingham, WA, 2005)

0277-786X/05/\$15 - doi: $10.1117 / 12.634088$

Proc. of SPIE 59250C-1 


\section{POLARISED STIMULATED EMISSION DEPLETION}

A schematic representation of the two-photon STED process in rhodamine 6G is shown in Figure 1. Initial excitation from low lying vibrational levels in the $S_{0}$ ground state occurs via the simultaneous absorption of two (non-resonant) near infra red photons with fast radiationless conversion yielding a vibrationally excited population in the $S_{1}$ excited electronic state. Fast collisional (and solvent) relaxation leads to the rapid population of lower vibrational levels in $S_{1}$. In the absence of external perturbations the population in $S_{1}$ decays by spontaneous emission to upper vibrational levels of $S_{0}$ followed by rapid collisional deactivation. In STED a visible laser pulse resonant with the $S_{1} \rightarrow S_{0}$ emission is applied to induce transitions to upper vibrational levels of $S_{0}$. The net result of this process is a sharp reduction in the excited state population (fluorescence intensity) and a change in molecular alignment (fluorescence anisotropy) dependent on the relative polarisation of the two laser pulses. Depletion of the excited state is maximised for parallel PUMP and DUMP polarisations and at PUMP-DUMP delays that are short in comparison to the rotational diffusion time of the molecule ${ }^{6-10}$. STED dynamics can be modelled analytically in the limit of fast ground state relaxation and weak depletion of the excited state population. For strong saturation of the DUMP transition analytic solutions to the coupled rate equations are possible for model DUMP pulse shapes ${ }^{6,7}$.
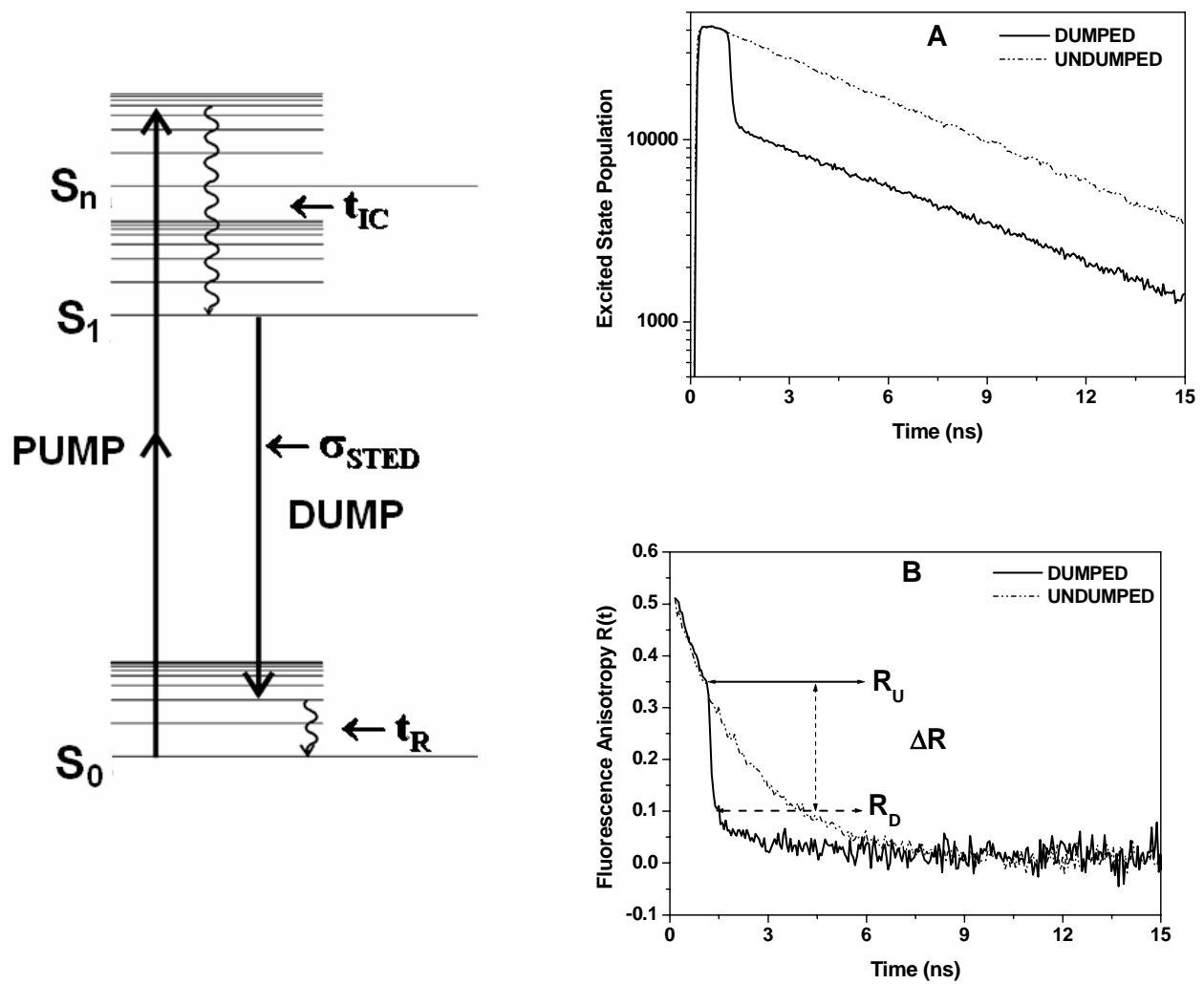

Figure 1: Stimulated emission depletion (STED) in rhodamine 6G. Linearly polarised two-photon excitation (PUMP) is followed by a time delayed (1ns, 17nJ, 622nm) DUMP pulse. With parallel PUMP and DUMP polarisations both population (A) and alignment (B) reductions are maximised. The reductions in the excited state population and alignment are $70 \%$ and 0.23 respectively 


\section{PHOTOSELECTION IN TWO-PHOTON EXCITATION}

\section{Excited State Orientational Distribution}

In experiments involving optical probes of molecular order and motion in fluid media it is advantageous to adopt a formalism in which the inherent symmetry of the radiation-molecule interaction and those of the experimental observables are fully exploited. To this end an expansion of the molecular orientational distribution function in terms of spherical harmonics has been widely used ${ }^{11-13}$. In the polar coordinate system in Figure 1, the probability of finding a molecule with an orientation between the polar angles $\theta$ and $\theta+d \theta, \phi$ and $\phi+d \phi$ is given by

$$
P(\theta, \phi)=\sum_{K Q}\left\langle C_{K Q}\right\rangle Y_{K Q}(\theta, \phi)
$$

where the expansion coefficients $\left\langle C_{K Q}\right\rangle$ define the moments of the distribution and can be found from (1) using the orthogonality of spherical harmonics,

$$
\left\langle C_{K Q}\right\rangle=\int_{0}^{2 \pi} \int_{0}^{\pi} Y_{K Q}^{*}(\theta, \phi) P(\theta, \phi) \sin \theta d \theta d \phi
$$

The probability of finding a molecule in any orientation on the unit sphere is necessarily unity, thus

$$
\sum_{\mathrm{KQ}}\left\langle\mathrm{C}_{\mathrm{KQ}}\right\rangle \int_{0}^{2 \pi} \int_{0}^{\pi} \mathrm{Y}_{\mathrm{KQ}}^{*}(\theta, \phi) \sin \theta \mathrm{d} \theta \mathrm{d} \phi=\sum_{\mathrm{KQ}}\left\langle\mathrm{C}_{\mathrm{KQ}}\right\rangle \sqrt{4 \pi} \delta_{\mathrm{K} 0} \delta_{\mathrm{Q} 0}=1
$$

giving a constant population moment (or monopole) $\left\langle\mathrm{C}_{00}\right\rangle=1 / \sqrt{4 \pi}$ The orientational probability can also be expressed in terms of normalised moments, $\left\langle\alpha_{\mathrm{KQ}}\right\rangle=\left\langle\mathrm{C}_{\mathrm{KQ}}\right\rangle /\left\langle\mathrm{C}_{00}\right\rangle$

$$
P(\theta, \phi)=\left[1+\sqrt{4 \pi} \sum_{K\rangle 0, Q}\left\langle\alpha_{K Q}\right\rangle Y_{K Q}(\theta, \phi)\right] / 4 \pi
$$

The multipole moments $\left\langle\alpha_{K Q}\right\rangle$ are directly related to measurable experimental quantities; moreover their transformation properties upon a change in coordinate system are particularly suited to fluorescence polarisation experiments ${ }^{14,15}$. In the weak excitation limit with a short pulsed optical excitation in an isotropic medium the moments of the initial excited state distribution are given by,

$$
\left\langle\alpha_{K Q}\right\rangle=\left[\int_{0}^{2 \pi} d \phi \int_{0}^{\pi} Y_{K Q}(\theta, \phi)^{*} W_{E X}(\theta, \phi) \sin \theta d \theta\right] /\left[\int_{0}^{2 \pi} d \phi \int_{0}^{\pi} Y_{0 o}(\theta, \phi)^{*} W_{E X}(\theta, \phi) \sin \theta d \theta\right]
$$

where $W_{E X}(\theta, \phi)$ is the angle dependent transition probability in the laboratory frame. The transition probability for the absorption of two photons of polarisations $\alpha$ and $\beta$ between states $i$ and $f$ is proportional to the squared modulus of the tensor $W_{\alpha \beta}^{i \rightarrow f}$ defined by a sum of transitions involving intermediate (virtual) states $\mathrm{n}^{16}$

$$
W_{E X}^{T P A}(\theta, \phi) \equiv\left|W_{\alpha \beta}^{i \rightarrow f}\right|^{2}=\left|\sum_{n}\left(\frac{\alpha \cdot\langle i|r| n\rangle\langle n|r| f\rangle \cdot \beta}{v_{n}-v_{\alpha}+i \Gamma_{n}}+\frac{\beta \cdot\langle i|r| n\rangle\langle n|r| f\rangle \cdot \alpha}{v_{n}-v_{\beta}+i \Gamma_{n}}\right)\right|^{2}
$$

where $v_{n}$ and $\Gamma_{n}$ are the transition frequency and homogeneous linewidth of $n$ respectively. In the molecular frame of reference the transition tensor can have 9 elements $\mathrm{S}_{\mathrm{AB}}$ where $\mathrm{A}, \mathrm{B}=\mathrm{X}, \mathrm{Y}$ or $\mathrm{Z}$. 


$$
\left|W_{\alpha \beta}^{i \rightarrow f}\right|^{2} \equiv\left|\sum_{A, B=X, Y, Z} S_{A B}\right|^{2}
$$

For planar aromatic molecules the two-photon transition is dominated by moments in the plane of the molecule ${ }^{17-19}$ and the summation over $Z$ can be neglected. For degenerate two-photon absorption with linearly polarised light $S_{\mathrm{AB}}=\mathrm{S}_{\mathrm{BA}}$ and $\alpha=\beta$. Photoselection in the laboratory frame is necessarily cylindrically symmetric about $\alpha$ and the excited state multipole moments are restricted to $\mathrm{Q}=0$. Further symmetry and angular momentum constraints inherent in the matrix elements of (6) restrict the rank $K$ to even integers with a maximum rank of $K=4$. As a result the initial excited state distribution function is characterised solely by $\left\langle\alpha_{20}\right\rangle$ and $\left\langle\alpha_{40}\right\rangle$

$$
P(\theta, \phi, t=0)=\frac{\left[Y_{00}(\theta, \phi)+\left\langle\alpha_{20}(0)\right\rangle Y_{20}(\theta, \phi)+\left\langle\alpha_{40}(0)\right\rangle Y_{40}(\theta, \phi)\right]}{\sqrt{4 \pi}}
$$

Where the transition is largely dominated by $S_{X X}$ the laboratory frame transition probability approximates to $\cos ^{4} \theta$ where $\theta$ is the angle between the laboratory $\mathrm{Z}$ axis and the $\mathrm{X}$ axis of the transition tenor which is taken to be the direction of the emission transition dipole moment. Under these circumstances (8) becomes

$$
P(\theta, \phi, t=0)=\frac{1}{\sqrt{4 \pi}}\left[Y_{00}(\theta, \phi)+\frac{4 \sqrt{5}}{7} Y_{20}(\theta, \phi)+\frac{8}{21} Y_{40}(\theta, \phi)\right]
$$

Polar plots of the angular contributions to $P(\theta, \phi)$ are shown in figure 2.

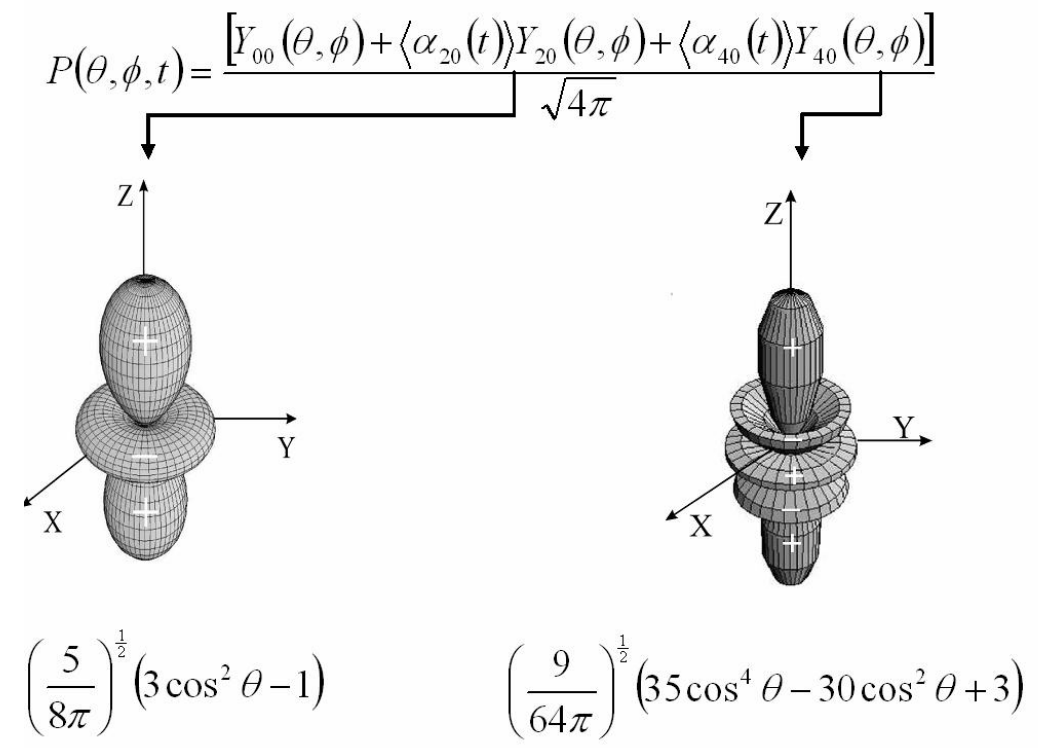

Figure 2: Angular contributions to the excited state orientational distribution prepared by two-photon excitation. 
The parallel and perpendicular components of the fluorescence anisotropy are given by

$$
\begin{aligned}
& I_{\|}(t) \propto N_{e x}(t=0) \int_{0}^{2 \pi} \int_{0}^{\pi} P(\theta, \phi, t=0) \cos ^{2} \theta \sin \theta d \theta d \phi \\
& I_{\perp}(t) \propto N_{e x}(t=0) \int_{0}^{2 \pi \pi} \int_{0}^{\pi} P(\theta, \phi, t=0) \sin ^{2} \theta \cos ^{2} \phi \sin \theta d \theta d \phi
\end{aligned}
$$

Expressing the parallel and perpendicularly polarised emission transition probabilities in terms of spherical harmonics yields

$$
\begin{aligned}
& I_{\|}(t=0) \propto N_{e x}(t=0) \int_{0}^{2 \pi} \int_{0}^{\pi} P(\theta, \phi, t=0)\left[Y_{00}(\theta, \phi)+\frac{2}{\sqrt{5}} Y_{20}(\theta, \phi)\right] \sin \theta d \theta d \phi \\
& I_{\perp}(t=0) \propto N_{e x}(t=0) \int_{0}^{2 \pi} \int_{0}^{\pi} P(\theta, \phi, t=0)\left[\begin{array}{l}
Y_{00}(\theta, \phi)-\frac{1}{\sqrt{5}} Y_{20}(\theta, \phi) \\
+\left(\frac{3}{10}\right)^{\frac{1}{2}}\left(Y_{22}(\theta, \phi)+Y_{2-2}(\theta, \phi)\right)
\end{array}\right] \sin \theta d \theta d \phi
\end{aligned}
$$

From the orthogonality of the spherical harmonics only the $\mathrm{K}=2$ rank moments of the excited state distribution can contribute to $I_{\|}$and $I_{\perp}$ and the initial fluorescence anisotropy becomes,

$$
R(0)=\frac{I_{\|}(t)-I_{\perp}(t)}{I_{\|}(t) 2 I_{\perp}(t)}=\frac{\left\langle\alpha_{20}(0)\right\rangle}{\sqrt{5}}=\frac{4}{7}
$$

For a molecular array with $100 \%$ alignment $P(\theta, \phi)=\delta_{\theta 0} \delta_{\phi 0}$ and the moments of the distribution function are given by

$$
\left\langle\alpha_{K Q}{ }^{M A X}\right\rangle=\sqrt{(2 K+1)} \delta_{Q 0}
$$

The maximum permissible values of $\left\langle\alpha_{20}\right\rangle$ and $\left\langle\alpha_{40}\right\rangle$ are $\sqrt{5}$ and 3 respectively.

\section{TWO-PHOTON FLUORESCENCE ANISOTROPY}

\section{Homogeneous Isotropic Fluid Media}

In isotropic fluid media molecular alignment following short pulse excitation is, in the case of small step rotational diffusion about a single molecular axis, governed by Debye diffusion dynamics ${ }^{20}$

$$
\frac{\partial P(\theta, \phi, t)}{\partial t}=D \nabla^{2} P(\theta, \phi, t)
$$

where $\mathrm{D}$ is the diffusion coefficient for the molecule. Inserting (1) into 14 yields

$$
\sum_{K Q} \frac{\partial}{\partial t}\left\langle C_{K Q}(t)\right\rangle Y_{K Q}(\theta, \phi)=\sum_{K Q}\left\langle C_{K Q}(t)\right\rangle D \nabla^{2} Y_{K Q}(\theta, \phi)
$$

The spherical harmonics $Y_{K Q}(\theta, \phi)$ are eigenfunctions of $\nabla^{2}$ with eigenvalues $-K(K+1)$ 


$$
\sum_{K Q} \frac{\partial}{\partial t}\left\langle C_{K Q}(t)\right\rangle Y_{K Q}(\theta, \phi)=-\sum_{K Q}\left\langle C_{K Q}(t)\right\rangle D K(K+1) Y_{K Q}(\theta, \phi)
$$

Multiplying by $Y_{K^{\prime} Q^{\prime}}(\theta, \phi)^{*}$ and integrating over the unit sphere (16) becomes

$$
\frac{\partial}{\partial t}\left\langle C_{K Q}(t)\right\rangle=-D K(K+1)\left\langle C_{K Q}(t)\right\rangle
$$

For small-step rotational diffusion to an isotropic steady state, the time dependence of $P(\theta, \phi)$ is thus

$$
P(\theta, \phi, t)=\frac{1}{\sqrt{4 \pi}}\left[Y_{00}(\theta, \phi)+\left\langle\alpha_{20}(t=0)\right\rangle \exp (-6 D t) Y_{20}(\theta, \phi)+\left\langle\alpha_{40}(t=0)\right\rangle \exp (-20 D t) Y_{40}(\theta, \phi)\right]
$$

From (12) the time-dependent fluorescence anisotropy is thus

$$
R(t)=\left(\left\langle\alpha_{20}(t=0)\right\rangle / \sqrt{5}\right) \exp (-6 D t) \equiv R(0) \exp \left(-t / \tau_{20}\right)
$$

The $\mathrm{K}=4$ orientational dynamics do not contribute to $R(t)$ but, as will be seen, the presence of hexadecapole alignment plays a significant role in stimulated emission dynamics. From (18) and (19) it is possible to predict the relative decay of the hexadecapole alignment from the change in fluorescence anisotropy,

$$
R(t) / R(0)=\exp \left(-t / \tau_{20}\right), \quad\left\langle\alpha_{40}(t)\right\rangle /\left\langle\alpha_{40}(0)\right\rangle=\exp \left(-10 t / 3 \tau_{20}\right)
$$

which yields

$$
\left[\left\langle\alpha_{40}(t)\right\rangle /\left\langle\alpha_{40}(0)\right\rangle\right]=[R(t) / R(0)]^{\frac{10}{3}}
$$

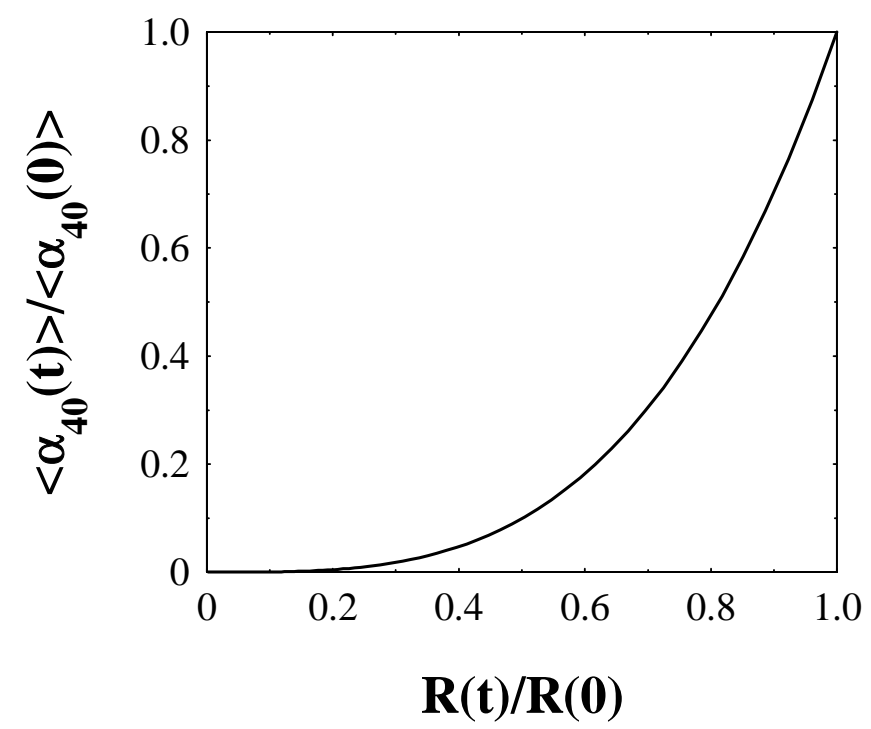

Figure 3: Variation in hexadecapole alignment with the decay of molecular alignment (fluorescence anisotropy) assuming small step isotropic rotational diffusion. 


\section{Rotational Diffusion in Liquid Crystals}

Compared to the single exponential decay that characterises single axis probe diffusion in homogeneous isotropic liquids, in liquid crystals the fluorescence anisotropy whilst decaying to zero (the medium being globally isotropic) is characterised by fast but restricted (incomplete) intra-pseudodomain rotational diffusion $\left(\tau_{20}{ }^{\mathrm{F}}\right)$ mediated by a slower overall orientational averaging $\left(\tau_{20} \mathrm{~S}\right)$ of the pesudodomain structure ${ }^{21}$. This behaviour has been observed in both single and two-photon fluorescence anisotropy studies of fluorescent probe motion in the isotropic phase of $5 \mathrm{CB}^{22}$. Interpretation of the probe anisotropy decays can be undertaken using the model of restricted rotational diffusion within a cone from which a local order parameter $\left\langle\alpha_{20}^{M}\right\rangle$ can be extracted.

$$
R(t) \cong R(0) \exp \left(-t / t_{20}^{S}\right)\left[\left(1-\left(\left\langle\alpha_{20}^{M}\right\rangle / \sqrt{5}\right)^{2}\right) \exp \left(-t / t_{20}^{F}\right)+\left(\left\langle\alpha_{20}^{M}\right\rangle / \sqrt{5}\right)^{2}\right]
$$

In such instances Debye diffusion does not hold and the simple relationship between $\left\langle\alpha_{20}\right\rangle$ and $\left\langle\alpha_{40}\right\rangle$ evolution as expressed in (17)-(21) and illustrated in figure 4 does not hold. In a highly structured local environment the divergence in $\tau_{40}{ }^{\mathrm{F}}$ and $\tau_{20}{ }^{\mathrm{F}}$ from (18) should be marked.

\section{POPULATION AND ALIGNMENT DYNAMICS IN STED}

\section{Weak Depletion Limit}

The contribution of the degrees of excited state alignment to STED observables can be highlighted in the limit of weak excited state depletion and fast ground state relaxation. The relationship between the initial (undumped) and final (dumped) orientational probability distribution functions being given by ${ }^{7}$,

$$
P_{D}(\theta, \phi)=\frac{N_{U}}{N_{D}} P_{U}(\theta, \phi)\left[1-S W_{D}(\theta, \phi)\right]
$$

$P_{U}(\theta, \phi), N_{U}, N_{D}$ and $P_{D}(\theta, \phi)$ are respectively the undumped (initial) and dumped (final) excited state populations and orientational distribution functions, $W_{D}(\theta, \phi)$ is the angular dependent DUMP transition probability and $S$ is the saturation parameter for the transition given by $S=\sigma E / h v A$ where $\sigma$ is the stimulated emission cross-section, $A$ is the beam area, $E$ and $h v$ are the DUMP pulse and photon energies respectively. Expressing the orientational distribution functions as in (1), the moments of the altered distribution for parallel PUMP and DUMP polarisations are

$$
\left\langle C_{K Q}^{D}\right\rangle=\frac{N_{U} \sqrt{4 \pi}}{3 N_{D}} \sum_{K^{\prime} Q^{\prime}}\left\langle C_{K^{\prime} Q^{\prime}}^{U}\right\rangle\left\langle K Q\left((3-S) Y_{00}(\theta, \phi)+\frac{2}{\sqrt{5}} S Y_{20}(\theta, \phi)\right) \mid K^{\prime} Q^{\prime}\right\rangle
$$

The superscripts $U$ and D refer respectively to the distributions immediately prior to and after the application of the DUMP pulse. Evaluation of the matrix elements for the directly observable moments of the altered array $\left\langle C_{00}^{D}\right\rangle$ and $\left\langle C_{20}^{D}\right\rangle^{7}$ yields analytic solutions for the fractional population removal $F_{D}$ and the change in the fluorescence anisotropy $\Delta R$

$$
\begin{gathered}
F_{D}=S\left(1+2 R_{U}\right) / 3 \\
\Delta R=R_{U}-R_{D}=S\left[\frac{2}{5}+\frac{4}{7} R_{U}-2 R_{U}{ }^{2}+\frac{12}{35}\left\langle\alpha_{40}^{U}\right\rangle\right] /\left[3-\left(1+2 R_{U}\right) S\right]
\end{gathered}
$$

Here $\left\langle\alpha_{40}^{U}\right\rangle$ and $R_{U}$ are respectively the normalised degree of hexadecapole alignment and the fluorescence anisotropy prior to excited state depletion. From (25) and (26) it can be seen that to first order the excited state depopulation 
depends solely on the incident photon flux (via $S$ ) and the initial degree of alignment (via $R_{U}$ ), the depolarisation of the excited state additionally depends on the hexadecapolar degree of excited state alignment. In STED experiments involving significant excited state depopulation and depolarisation the departure from first order behaviour is marked. Nonetheless as will be seen $\Delta R$ saturation dynamics are considerably more sensitive to hexadecapolar alignment than the variation in $F_{D}$ with DUMP pulse energy.

\section{Rate Equation Analysis}

The rate equations for STED on a timescale that is fast compared to spontaneous emission (nanosecond to subnanosecond) and orientational relaxation (nanosecond) are given by ${ }^{7}$

$$
\begin{aligned}
& \frac{d N_{E X}(\theta, \phi, t)}{d t}=-\frac{I_{D}(t) \sigma W_{D}(\theta, \phi)}{h v}\left(N_{E X}(\theta, \phi, t)-N_{G S}(\theta, \phi, t)\right) \\
& \frac{d N_{G S}(\theta, \phi, t)}{d t}=\frac{I_{D}(t) \sigma W_{D}(\theta, \phi)}{h v}\left(N_{E X}(\theta, \phi, t)-N_{G S}(\theta, \phi, t)\right)-\frac{N_{G S}(\theta, \phi, t)}{t_{R}}
\end{aligned}
$$

Where $N_{E X}(\theta, t)$ and $N_{G S}(\theta, t)$ are the ground and excited state populations, $I_{D}(t)$ is the DUMP pulse intensity, $\mathrm{W}_{\mathrm{D}}(\theta)$ is the anglular dependence of the DUMP probability, $\sigma$ is the stimulated emission cross-section $\left(\mathrm{cm}^{2}\right)$ and $t_{\mathrm{R}}$ is the ground state vibrational relaxation time. It is possible to obtain analytic solutions to the rate equations by assuming a rectangular DUMP pulse with width $t_{P}$, which yield the fractional remaining population $F_{R}$ as a function of $E_{P} \sigma / h v A$ for given ratios of $t_{P} / t_{R}$. Given that the initial population distribution in the upper ground state vibrational levels is negligible, the excited state population evaluated at $t=t_{P}$ is

$$
N_{E X}\left(\theta, \phi, t_{P}\right)=\frac{N_{E X}(\theta, \phi, 0)}{2 d} \exp \left(-\frac{1}{2}\left\{\frac{t_{P}}{t_{R}}+2 S \cos ^{2} \theta+d\right\}\right)\left[\frac{t_{P}}{t_{R}}\left(e^{d}-1\right)+d\left(e^{d}+1\right)\right]
$$

where the parameter $\mathrm{d}$ is given by

$$
d=\sqrt{\left(t_{P} / t_{R}\right)^{2}+4 S^{2} \cos ^{4} \theta}
$$

The fractional depletion of the excited state population $F_{D}$ and the anisotropy change $\Delta R$ can be calculated from

$$
\begin{gathered}
F_{D}=\int_{0}^{2 \pi} \int_{0}^{\pi}\left[N_{E X}(\theta, \phi, 0)-N_{E X}\left(\theta, \phi, t_{P}\right)\right] \sin \theta d \theta d \phi / \int_{0}^{2 \pi \pi} \int_{0}^{\pi} N_{E X}(\theta, \phi, 0) \sin \theta d \theta d \phi \\
\Delta R=\int_{0}^{2 \pi \pi} \int_{0}\left[N_{E X}(\theta, \phi, 0)-N_{E X}\left(\theta, \phi, t_{P}\right)\right] \frac{1}{2}\left(3 \cos ^{2} \theta-1\right) \sin \theta d \theta d \phi / \int_{0}^{2 \pi} \int_{0}^{2 \pi} N_{E X}(\theta, \phi, 0) \sin \theta d \theta d \phi
\end{gathered}
$$

where

$$
N_{E X}(\theta, \phi, 0)=\frac{1}{\sqrt{4 \pi}}\left[Y_{00}(\theta, \phi)+R_{U} \sqrt{5} Y_{20}(\theta, \phi)+\left\langle\alpha_{40}^{U}\right\rangle Y_{40}(\theta, \phi)\right]
$$

Anisotropy and population saturation curves can be generated from (29)-(33) using a Mathematica programme, for varying $\mathrm{S}$ and ratios of DUMP pulse-width to ground state vibrational relaxation time $\left(\mathrm{t}_{\mathrm{p}} / \mathrm{t}_{\mathrm{R}}\right)$ with $R_{U}($ known $)$ and $\left\langle\alpha_{40}^{U}\right\rangle$ as inputs. 


\section{EXPERIMENTAL PROCEDURE}

The experimental apparatus for STED has been described in detail elsewhere ${ }^{7,8,10}$. Two experimental geometries are employed; studies of Rhodamine $6 \mathrm{G}$ in ethylene glycol were carried out using a $90^{\circ}$ excitation-detection geometry (figure 4A). A collinear $\left(180^{\circ}\right)$ excitation-detection geometry (figure 4B) was employed for STED measurements in 5CB which were undertaken in $75 \mu \mathrm{m}$ path length cells. Time-resolved fluorescence lifetime and anisotropy measurements at fixed PUMP-DUMP delays were performed using picosecond photon counting. Excited state population depletion measurements were obtained from dumped and undumped total fluorescence intensity decays. Fine adjustments to the PUMP and DUMP energies were made using neutral density wheels (Melles-Griot). The typical on-sample PUMP pulse energies were below 30nJ, the DUMP pulse energy was varied between 0 and 33nJ. A DUMP wavelength of $645 \mathrm{~nm}$ was selected to minimise the effects of DUMP induced fluorescence in rhodamine $6 \mathrm{G}$ and $5 \mathrm{CB}$.

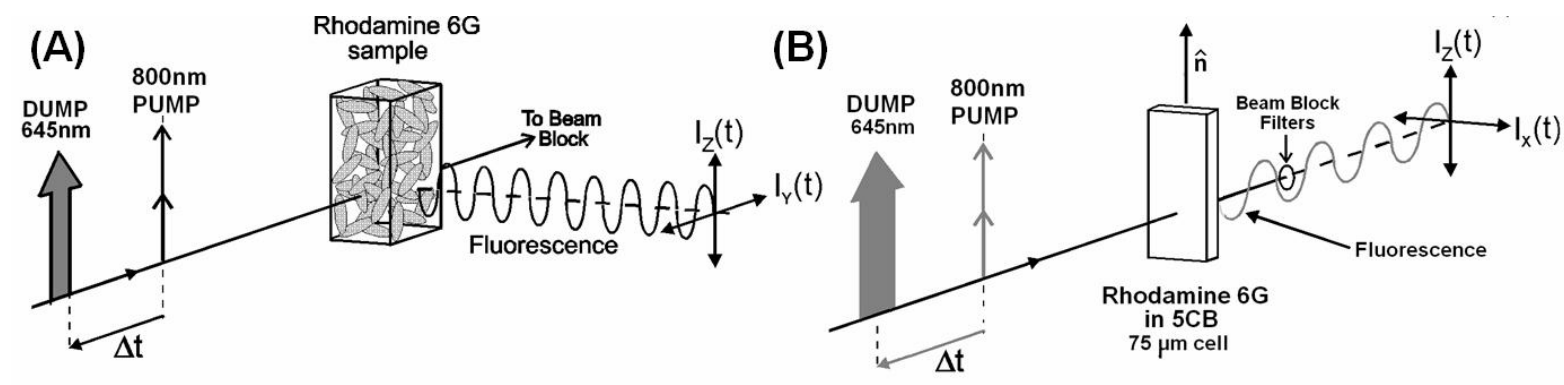

Figure 4: Excitation-detection geometries employed in the time-resolved stimulated emission depletion experiments.

\section{RESULTS}

Fluorescence depletion and depolarisation measurements of rhodamine $6 \mathrm{G}$ in ethylene glycol were undertaken using a PUMP-DUMP delay of 600ps, corresponding to an initial anisotropy $R_{U}$ of 0.41 . The maximum DUMP pulse energy was $33 \mathrm{~nJ}$; the variation in $F_{D}$ and $\Delta R$ are displayed in figure 5. Fits to the data setting $\left\langle\alpha_{40}^{U}\right\rangle=0$ whilst yielding an apparently reasonable agreement to the $F_{D}$ data $(5 \mathrm{~A} 1)$ do not reproduce the observed energy dependence of $\Delta R$ (5A2). Values of $\left\langle\alpha_{40}^{U}\right\rangle$ were increased with the $F_{D}$ and $\Delta R$ data best fit by $\left\langle\alpha_{40}^{U}\right\rangle=0.35$ and $\mathrm{t}_{\mathrm{P}} / \mathrm{t}_{\mathrm{R}}=3(5 \mathrm{~B} 1 \& 5 \mathrm{~B} 2$ ). For rhodamine $6 \mathrm{G}$ in $5 \mathrm{CB}$ a PUMP DUMP delay of 650ps was employed corresponding to an initial anisotropy $R_{U}$ of 0.42 . Measurements of $F_{D}$ and $\Delta R$ up maximum DUMP energy of c.a. $19 \mathrm{~nJ}$ were performed and the results displayed in figure 6 . As with the saturation data for rhodamine $6 \mathrm{G}$ in ethylene glycol, setting $\left\langle\alpha_{40}^{U}\right\rangle=0$ does not lead to a satisfactory fit to the $\Delta R$ data. Values of $\left\langle\alpha_{40}^{U}\right\rangle$ were increased for both $F_{D}$ and $\Delta R$ simulations until an optimum value was achieved. The results are shown in figure 6 where an initial hexadecapole alignment of 0.6 provides the best fit to both $F_{D}$ and $\Delta R$ data, with the best value of $t_{\mathrm{P}} / \mathrm{t}_{\mathrm{R}}$ close to 1.5 . In ethylene glycol and $5 \mathrm{CB}$, DUMP pulsewidths of $1 \mathrm{ps}$ and $1.5 \mathrm{ps}$ were employed corresponding to ground state relaxation times of $333 \mathrm{fs}$ and $1 \mathrm{ps}$ respectively.

For rhodamine $6 \mathrm{G}$ in ethylene glycol the initial degree of photoselected hexadecapole alignment can be predicted from fluorescence anisotropy data via (21), these together with the predictions based on the assumption of a single element diagonal transition tensor are shown in table 1. For Rhodamine $6 \mathrm{G}$ predictions of the initial hexadecapole alignment based on the change in fluorescence anisotropy yield an initial value for $\left\langle\alpha_{40}\right\rangle$ of 0.77 , this is considerably larger than the theoretical value of 0.38 that would be expected for a single element transition tensor. Recent circular and linearly polarised two-photon fluorescence anisotropy and absorption anisotropy measurements ${ }^{23}$ indicate that the rhodamine $6 \mathrm{G}$ transition tensor has the form

$$
S=\left[\begin{array}{ll}
S_{X X} & S_{X Y} \\
S_{Y X} & S_{Y Y}
\end{array}\right] \propto\left[\begin{array}{cc}
1 & |0.1171| \\
|0.1171| & 0.1086
\end{array}\right]
$$



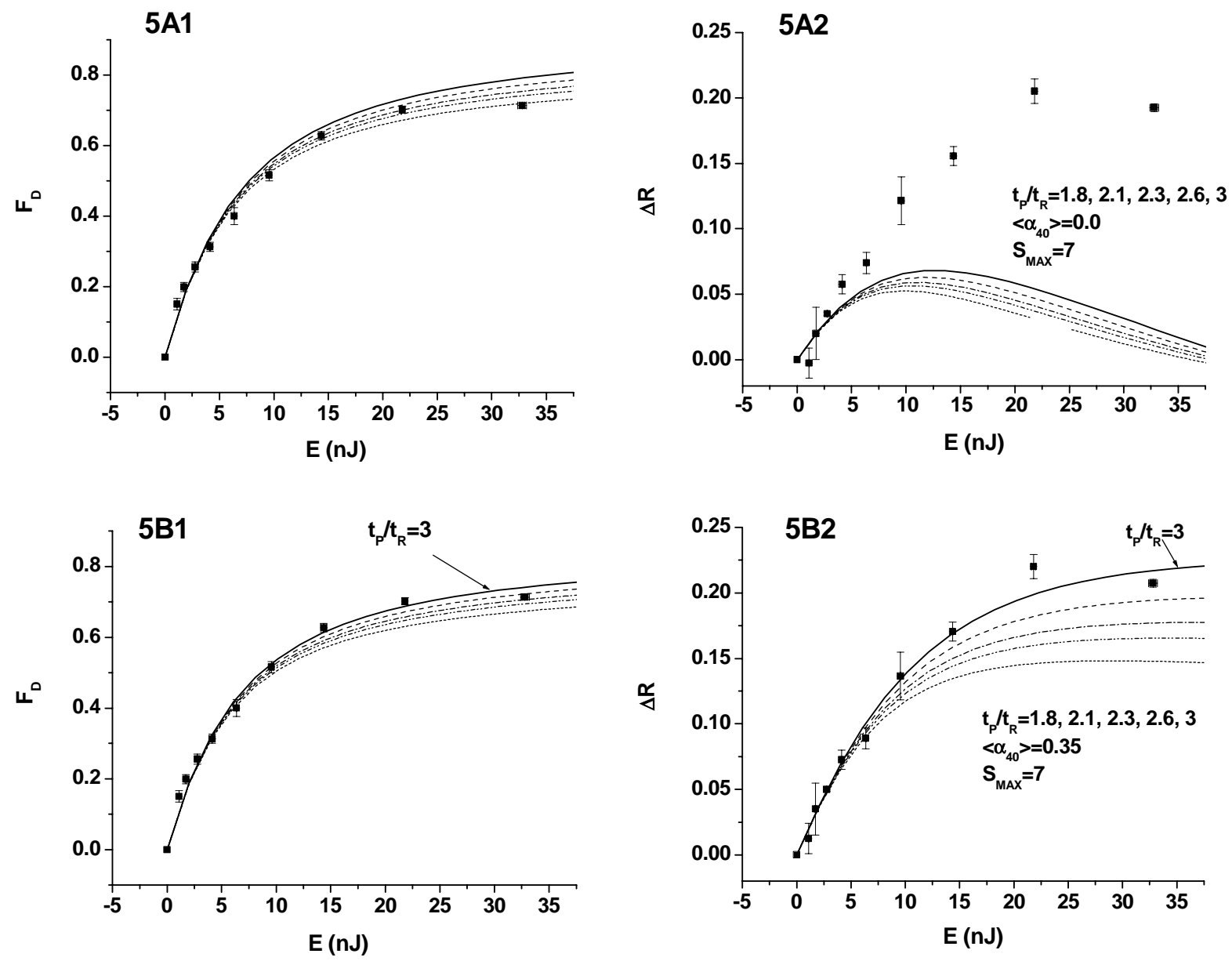

Figure 5 Excited state depopulation $\left(F_{D}\right)$ and depolarisation measurements for rhodamine $6 G$ in ethylene glycol, the PUMP-DUMP separation was 600ps corresponding to an initial anisotropy $R_{U}$ of 0.41. A1 and A2 show fits to the data neglecting the contribution of hexadecapole alignment, B1 and B2 show fits to the data with $\left\langle\alpha_{40}^{U}\right\rangle=0.35$, the best agreement between $F_{D}$ and $\Delta R$ data is given by $t_{P} / t_{R}=3$. 

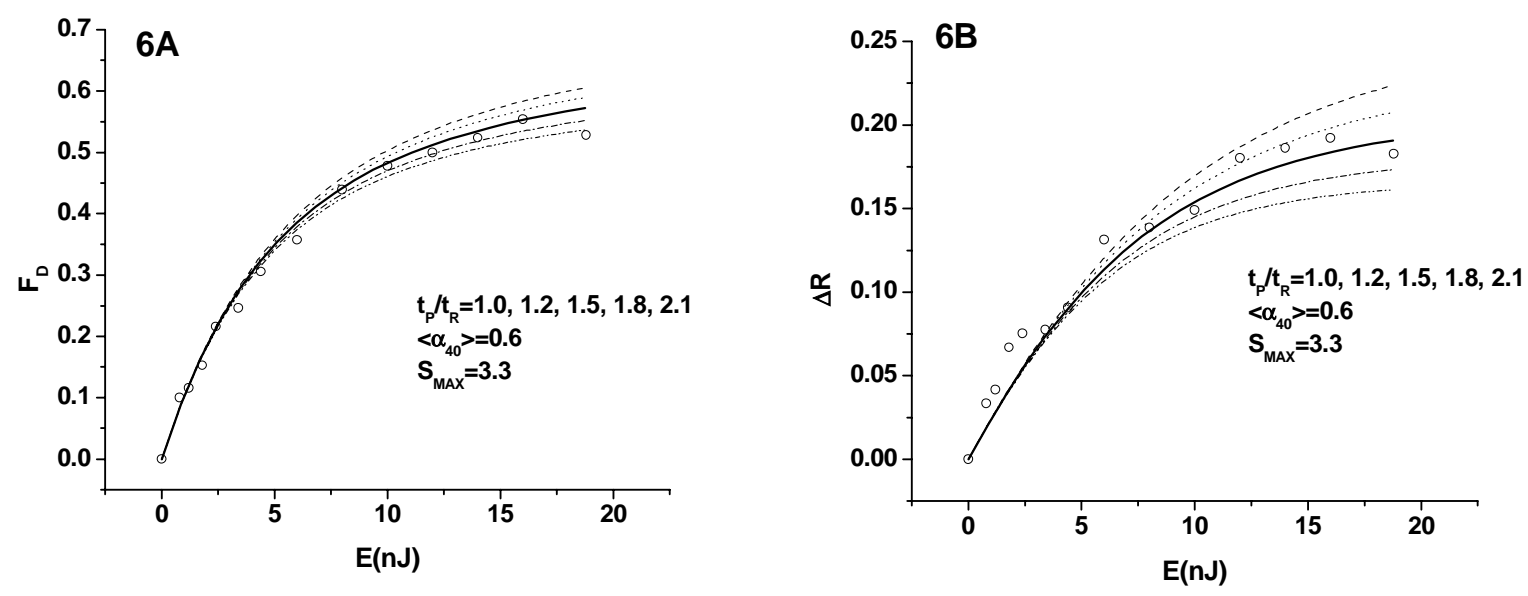

Figure $6(A)$ Excited state depopulation $\left(F_{D}\right)$ and $(B)$ depolarisation measurements $\Delta R$ for rhodamine $6 G$ in the isotropic phase of $5 C B$. The PUMP-DUMP separation was 600ps corresponding to an initial anisotropy $R_{U}$ of $0.42 . T$ The $F_{D}$ and $\Delta R$ data is best fit by simulations in which the undumped hexadecapole alignment is $=0.6$ and $t_{P} / t_{R}=1.5$.

The divergence in the degree of photoselected hexadecapole alignment from that predicted by a single element transition tensor $\left(S_{i j}=\delta_{i X} \delta_{j X}\right)$ has yet to be calculated although initial degrees of alignment greater than $(4 \sqrt{ } 5) / 7$ have been shown to arise from interference terms in of different $\operatorname{sign}^{16,18,19}$. In the case of rhodamine $6 \mathrm{G}$ in $5 \mathrm{CB}$ the value of $\left\langle\alpha_{40}^{U}\right\rangle$ at $650 \mathrm{ps}$ after excitation is 0.6. Hexadecapole alignment dynamics in a medium exhibiting bi-exponential rotational diffusion cannot be related to fluorescence anisotropy changes via simple relationships such as (21). Here predictions of $\left\langle\alpha_{40}\right\rangle$ evolution require knowledge of both the local steady-state hexadecapole alignment and the relationship between the $\mathrm{K}=2$ and $\mathrm{K}=4$ domain and intra-domaim relaxation times. Nonetheless without the assumption of any detailed model for the higher order diffusion dynamics it is clear that for a comparable degree of excited state alignment to that in ethylene glycol the degree of hexadecapole alignment that is present in the array 600ps after excitation is considerably larger than in ethylene glycol. If the initial value of the hexadecapole alignment is 0.77 for both molecules (i.e. solvent independent) then it is clear that hexadecapole alignment relaxation in 5CB is considerably slower than the alignment dynamics. This is somewhat surprising as the sensitivity (i.e. decay) of a higher order correlation function to angular averaging should be greater. The anisotropy of the rhodamine $6 \mathrm{G}$ transition tensor in $5 \mathrm{CB}$ is currently under investigation in our laboratory.

\begin{tabular}{|l|c|c|c|c|c|}
\hline & $\begin{array}{c}\text { Initial } \\
\text { Anisotropy R(0) }\end{array}$ & $\begin{array}{c}\text { Undumped } \\
\text { Anisotropy } \mathbf{R}_{\mathrm{U}}\end{array}$ & $\left\langle\alpha_{40} \mathrm{U}\right\rangle$ & $\left.<\alpha_{40}(\mathbf{0})\right\rangle$ & $\begin{array}{c}\left.<\alpha_{40}(\mathbf{0})\right\rangle \\
\left(S_{X X}\right. \\
\text { tensor })\end{array}$ \\
\hline $\begin{array}{l}\text { Rhodamine 6G } \\
\text { (ethylene glycol) }\end{array}$ & $\mathbf{0 . 5 2}$ & $\mathbf{0 . 4 1}$ & $\mathbf{0 . 3 5}$ & $\mathbf{0 . 7 7}$ & $\mathbf{0 . 3 8}$ \\
\hline Rhodamine 6G in 5CB & $\mathbf{0 . 4 9 7}$ & $\mathbf{0 . 4 2}$ & $\mathbf{0 . 6 0}$ & $\mathbf{( 1 . 0 5 )}$ & $\mathbf{0 . 3 8}$ \\
\hline
\end{tabular}

Table 1 Initial fluorescence anisotropies $(R(0))$, and undumped fluorescence anisotropies $\left(R_{U}\right)$ for rhodamine $6 G$ in ethylenc glycol and $5 C B$, together with the calculated undumped hexadecapole alignment, the initial hexadecapole alignment is extrapolated assuming Debye diffusion dynamics; the value of 1.07 for 5CB is in brackets as Debye diffusion dynamics are not obeyed in this system. 


\section{CONCLUSIONS}

Stimulated emission population and depolarisation measurements permit the measurement of the previously hidden degrees of excited state alignment that are created by two-photon excitation. Measurements of the degree of hexadecapole order in rhodamine 6G yield values that are substantially larger than those expected for a single element transition tensor $\left(S_{X X}\right)$. The presence of off diagonal elements is known to yield values of the initial molecular alignment in excess of that obtained for $S_{X X}$ alone; our results indicate that the creation of hexadecapole alignment is a highly sensitive probe of such structure. Differences between hexadecapole and alignment relaxation dynamics of rhodamine $6 \mathrm{G}$ in the isotropic phase of $5 \mathrm{CB}$ are also apparent and are the subject of ongoing study.

\section{ACKNOWLEDGEMENTS}

We would like to thank EPSRC for support of this work.

\section{REFERENCES}

[1] D. E. Reisner, R. W. Field, J. L. Kinsey and H-L. Dai, J. Chem. Phys. 80 5968- 59781984.

[2] I. Gryczynski, J. Kusba and J. R. Lakowicz, J. Chem. Phys. 111 89-99 1994, J. Kusba, V. Bogdanov, I. Gryczynski and J. R. Lakowicz, Biophys. J. 67 2024-2040 1994

[3] S. A. Kovalenko, J. Ruthmann and N. P. Ernsting, J.Chem Phys. 109 1894-1900 1998

[4] Ch. Buehler, C. Y. Dong, P. T. C. So, T. French and E. Gratton. Biophys. J 79 536-549, 2000

[5] T. A. Klar, S. Jakobs, M. Byba, A. Egner and S. W. Hell Proc. Nat. Acad. Sci. (USA) 9782062000

[6] D. A Armoogum, A. J. Bain and R. J. Marsh, Proc. Int Soc. Opt. Eng. 4812 45-54 2002

[7] R. J. Marsh, D. A. Armoogum and A. J. Bain, Chem. Phys. Lett. 366 398-405 2002.

[8] D. A. Armoogum R. J. Marsh and A. J. Bain Proc. Int Soc. Opt. Eng. 5222 34-44 (2003)

[9] A. J. Bain, R. J. Marsh and D. A. Armoogum, L. Porres O. Mongin and M. Blanchard-Desce Bio. Chem. Soc Trans 31, 1047-1051 2003

[10] R. J. Marsh, N. D. Leonczek D. A. Armoogum L. Porres O. Mongin M. Blanchard-Desce and A. J. Bain Proc. Int Soc. Opt. Eng. 5510 117-128 2004

[11] T. Tao Biopolymers 8 609-632 1969

[12] C. Zannoni, A Arcioni and P Cavatorta Chem. Phys. Lipds 32: 179-250 1983

[13] A. Szabo J. Chem. Phys. 72 4620- 46261984.

[14] A. J. Bain, P. Chandna, and J. Bryant, J. Chem. Phys. 112, 10418-10434 2000

[15] A. J. Bain, P. Chandna, and J. Bryant, J. Chem. Phys. 112 10434-10449 2000

[16] C. Z. Wan and C. K. Johnson Chem. Phys. 179 513-531 1994

[17] P. R. Callis J. Chem .Phys. 99 27-37 1993

[18] S. W. Pauls, J. F. Hedstrom and C. K. Johnson, Chem. Phys. 237 205-222. 1998

[19] P. R. Callis Annu. Rev. Phys. Chem. 48 271-297 1997

[20] B. Berne and R. Pecora Dynamic Light Scattering (Wiley New York 1976)

[21] E. M. Monge J. Bryant B. Obradovic A. Harsono and A. J. Bain Proc. Int Soc. Opt. Eng. 5213 213-223 2003

[22] E. M. Monge PhD Thesis University of London 2003 (unpublished)

[23] R. J. Marsh, N. D. Leonczek D. A. Armoogum L. Porres O. Mongin M. Blanchard-Desce and A. J. Bain (to be published) 